\title{
Seroprevalence of Toxoplasma gondii infection in household and stray cats in Lanzhou, northwest China
}

\author{
Song-Ming $\mathrm{Wu}^{1,2}$, Xing-Quan Zhu1,3, Dong-Hui Zhou', Bao-Quan Fu', Jia Chen', Jian-Fa Yang ${ }^{3}$, Hui-Qun Song ${ }^{1}$,
} Ya-Biao Weng ${ }^{2}$ and De-He Ye 4 $^{*}$

\begin{abstract}
Background: Toxoplasma gondii is an important protozoan parasite infecting humans and almost all warmblooded animals. As the only definitive host, cats play a crucial role in the transmission of $T$. gondii infection by shedding parasite oocysts in their feces. However, little information on T. gondii infection in cats was available in Lanzhou, northwest China. This study was performed to determine the seroprevalence of $T$. gondii infection in household and stray cats in Lanzhou, northwest China.

Results: A total of 221 (179 households and 42 strays) blood samples were collected from clinically healthy cats admitted to several pet hospitals located in Lanzhou City, between November 2010 and July 2011 for the serological detection of $T$. gondii infection. The majority (207) of these cats represented Chinese Lihua cats. 47 of 221 (21.3\%) examined cats were seropositive for T. gondii infection using the modified agglutination test (MAT) at the cut-off of 1:25. The seroprevalence in household and stray cats was assessed to be $15.6 \%$ and $45.2 \%$, respectively, and the difference was statistically significant $(P<0.05)$. The seroprevalence ranged from $15.1 \%$ to $25.8 \%$ among different age groups, but the differences were not statistically significant $(P>0.05)$. Studies showed that there was no relationship between seroprevalence and the gender $(P>0.05)$.
\end{abstract}

Conclusions: The present survey indicated the high seroprevalence of T. gondii in cats in Lanzhou, northwest China, which poses a threat to animal and human health. Therefore, measures should be taken to control and prevent toxoplasmosis of cats in this area.

\section{Background}

Toxoplasma gondii is an obligate intracellular parasite, affecting humans and a wide range of warm-blooded animals worldwide [1-3]. T. gondii infection is a global concern, and about one third of the human population has been exposed to this parasite [1]. Toxoplasmosis is one of the most important food-borne diseases that can cause toxoplasmic encephalitis in immuno-compromised patients, blindness, abortion, fetal abnormalities or even prenatal death in congenital cases [3,4]. Humans or animals can acquire $T$. gondii infection post-natally by ingestion of undercooked or raw meat from infected animals, or ingestion of food or water contaminated

\footnotetext{
* Correspondence: gndydh@126.com

${ }^{4}$ College of Veterinary Medicine, Gansu Agricultural University, Lanzhou,

Gansu Province 730070, PR China

Full list of author information is available at the end of the article
}

with oocysts excreted by infected felids, or ingestion of oocysts from the environment by accident $[1,5]$.

Felids are considered the only definitive hosts of T. gondii playing a crucial role in the transmission of the parasite [6]. Cats infected by T. gondii may pose a potential threat to public health, because they can shed and excrete environmentally resistant oocysts in their feces $[7,8]$. Household cats are one of the most intimate companions of humans. By frequent contact with cats, people may increase their risk of acquiring T. gondii infection. More importantly, stray cats usually wander everywhere and play a more important role in the transmission of toxoplasmosis to other animals and humans [6].

Surveys of T. gondii infection in stray and household cats have been reported extensively in the world [1,9], including mainland China [10-13]. However, little is

\section{Ciomed Central}


known about the infection of $T$. gondii in stray and household cats in Lanzhou, northwest China. The objective of the present study was to determine the seroprevalence of $T$. gondii infection in stray and household cats in Lanzhou.

\section{Materials and methods}

\section{The investigated city}

The survey was conducted in Lanzhou City $\left(35^{\circ} 5^{\prime \prime} \sim 38^{\circ}\right.$ $\mathrm{N}, 102^{\circ} 30^{\prime \prime} \sim 104^{\circ} 30^{\prime \prime} \mathrm{E}$ ), which is the capital of Gansu province, covering an area of approximately 13, 000 square meters in northwest China. This city is at an elevation of approximately 1, 500 meters, crossed by the Yellow River from west to east, having a characteristic ribbon basin geography. The climate is temperate and continental monsoonal with an average annual temperature of $9.3^{\circ} \mathrm{C}$ and annual precipitation of $360 \mathrm{~mm}$.

\section{Naturally infected cats}

Between November 2010 and July 2011, a total of 221 blood samples were obtained from stray and household cats in Lanzhou. These clinically healthy cats were admitted into pet hospitals located in three districts of Lanzhou City, namely Chengguan District, Anning District and Xigu District, for the serological detection of $T$. gondii infection. Information regarding the breed, age, gender and geographical origin of pet cats were obtained from their owners, and the biometric data of stray cats were estimated based on body condition and dental age. Blood samples were kept at room temperature for $2 \mathrm{~h}$, centrifuged at 3,000 rpm for $5 \mathrm{~min}$, and the separated serum samples were stored at $-20^{\circ} \mathrm{C}$ until further analysis. This study was approved by the Animal Ethics Committee of Lanzhou Veterinary Research Institute, Chinese Academy of Agricultural Sciences.

\section{Serological examination}

Antibodies to $T$. gondii were determined in cat sera by the modified agglutination test (MAT) as described previously [14]. In this study, we chose MAT because it is sensitive and specific for detecting $T$. gondii antibodies in many animals as compared to other serologic methods [15-17]. In brief, sera were added to "U" bottom 96 well microtiter plates, diluted two-fold starting from $1: 25$ to $1: 1600$, the plates were shaken for $2 \mathrm{~min}$ and then incubated at $37^{\circ} \mathrm{C}$ overnight without shaking. Sera with MAT titers of 1:25 or higher were considered positive, and those sera with dubious results were re-tested. Positive and negative controls were incorporated in each test.

\section{Statistical analysis}

Differences in the seroprevalence of $T$. gondii infection between male and female cats, and among different age groups were analyzed using a Chi square test using the SPSS for Windows (Release 18.0 standard version, SPSS Inc., Chicago, Illinois). The differences were considered statistically significant when $P<0.05$.

\section{Results}

In this study, serum samples were obtained from a total of 211 clinically healthy cats (179 households and 42 strays). The majority (207) of these cats represented Chinese Lihua cats. 47 of 221 (21.3\%) examined cats were seropositive for $T$. gondii infection by MAT at the cut-off of 1:25 (Table 1). Seroprevalence of T. gondii infection in household and stray cats were $15.6 \%$ and $45.2 \%$, respectively. Among different age groups, the seroprevalence varied from $15.1 \%$ to $25.8 \%$ (Table 1 ).

The seroprevalence in male cats was $25 \%$, and in females it was $17.9 \%$ (Table 1 ). Table 2 shows the distribution of antibodies to T. gondii in household and stray

Table 1 Seroprevalence of Toxoplasma gondii infection in household and stray cats by gender and age in Lanzhou, northwest China using modified agglutination test (MAT)

\begin{tabular}{|c|c|c|c|c|c|c|c|c|c|}
\hline \multirow[t]{3}{*}{ Cat groups } & \multicolumn{6}{|c|}{ Types of cat } & \multicolumn{3}{|l|}{ Total } \\
\hline & \multicolumn{3}{|c|}{ Household cat } & \multicolumn{3}{|l|}{ Stray cat } & \multirow[b]{2}{*}{ No. tested } & \multirow[b]{2}{*}{ No. positive } & \multirow[b]{2}{*}{ Prevalence $(\%$} \\
\hline & No. tested & No. positive & Prevalence (\%) & No. tested & No. positive & Prevalence (\%) & & & \\
\hline \multicolumn{10}{|l|}{ Gender } \\
\hline Male & 87 & 17 & 19.5 & 17 & 9 & 52.9 & 104 & 26 & 25 \\
\hline Female & 92 & 11 & 12 & 25 & 10 & 40 & 117 & 21 & 17.9 \\
\hline \multicolumn{10}{|l|}{ Age (years) } \\
\hline$<1$ & 41 & 4 & 9.8 & 12 & 4 & 33.3 & 53 & 8 & 15.1 \\
\hline 2 & 65 & 12 & 18.5 & 24 & 11 & 45.8 & 89 & 23 & 25.8 \\
\hline 3 & 37 & 6 & 16.2 & 3 & 2 & 66.7 & 40 & 8 & 20 \\
\hline$\geq 3$ & 36 & 6 & 16.7 & 3 & 2 & 66.7 & 39 & 8 & 20.5 \\
\hline Total & 179 & 28 & 15.6 & 42 & 19 & 45.2 & 221 & 47 & 21.3 \\
\hline
\end{tabular}


Table 2 Antibody titers to Toxopalsma gondii infection in household and stray cats in Lanzhou, northwest China by modified agglutination test (MAT)

\begin{tabular}{|c|c|c|c|c|c|c|c|c|}
\hline \multirow{2}{*}{$\begin{array}{l}\text { Types of } \\
\text { cat }\end{array}$} & \multicolumn{8}{|c|}{ No. of sera with MAT titers of } \\
\hline & $1: 25$ & $1: 50$ & $1: 100$ & $1: 200$ & $1: 400$ & $1: 800$ & $\geq 1: 1600$ & Total \\
\hline $\begin{array}{l}\text { Household } \\
\text { cat }\end{array}$ & 3 & 5 & 5 & 1 & 1 & 2 & 11 & 28 \\
\hline Stray cat & 1 & 3 & 1 & 1 & 1 & 3 & 9 & 19 \\
\hline Total & 4 & 8 & 6 & 2 & 2 & 5 & 20 & 47 \\
\hline
\end{tabular}

cats determined using MAT, and antibody titers ranged from 1:25 to $1: 1600$ or higher. Table 3 shows the seroprevalence of $T$. gondii infection in household and stray cats in relation to their breeds.

\section{Discussion}

The overall prevalence of $T$. gondii infection in cats in Lanzhou was $21.3 \%$, which was lower than that reported in some other countries, such as in Iran (32.1\%) $[1,18]$, lower than that observed in Guangzhou City (25.2\%) [11], but higher than that in Zhengzhou City (15.5\%) and Beijing City (14.1\%) in China $[19,20]$. The differences in seroprevalences of $T$. gondii in cats are probably due to differences in ecological and geographical factors, serologic tests used and the living conditions for cats. In general, T. gondii oocysts are more likely to survive in warm and humid environments [1]. The warm and humid climate in southern China (such as Guangzhou) is favorable for the transmission of $T$. gondii, whereas the cold and dry climate in winter in Lanzhou may be less favorable for the spread of T. gondii.

The results indicated that prevalence of antibodies varied with ages, and $T$. gondii seroprevalence in older animals was generally higher than that in young animals, however, the differences were not statistically significant

\begin{tabular}{|c|c|c|c|}
\hline Breeds of cat & $\begin{array}{l}\text { No. } \\
\text { examined }\end{array}$ & $\begin{array}{l}\text { No. } \\
\text { positive }\end{array}$ & $\begin{array}{l}\text { Prevalence } \\
\text { (\%) }\end{array}$ \\
\hline Chinese Lihua cat & 207 & 45 & 21.7 \\
\hline Chausie cat & 1 & 0 & 0 \\
\hline Persian cat & 5 & 0 & 0 \\
\hline Russian Blue cat & 1 & 1 & 100 \\
\hline Dragen-Li cat & 2 & 0 & 0 \\
\hline British Shorthair cat & 2 & 1 & 50 \\
\hline Mainecoon cat & 1 & 0 & 0 \\
\hline $\begin{array}{l}\text { Highland Scottish Fold } \\
\text { cat }\end{array}$ & 2 & 0 & 0 \\
\hline Total & 221 & 47 & 21.3 \\
\hline
\end{tabular}

$(P>0.05)$. Female cats had lower prevalence than the male animals, although the difference was not significant $(P>0.05)$.

In this study, seroprevalence of $T$. gondii infection in stray cats was $45.2 \%$, which is significantly higher than that $(15.6 \%)$ in household cats $(P<0.05)$, consistent with reports from some other countries [21,22]. Differences in their hunting habits, living conditions and animal welfare may attribute to the difference in $T$. gondii seroprevalence between household cats and stray cats. Table 2 shows that $20(42.6 \%)$ of the 47 infected cats had anti-T. gondii titers of 1:1600 or higher, indicating that these cats suffered from severe infection and/or repeated exposure to $T$. gondii, shedding oocysts to the environment.

The present study examined seroprevalence of $T$. gondii infection in clinically healthy household and stray cats. Further investigations of $T$. gondii seroprevalence in diseased cats concurrently infected with other feline pathogens are warranted because previous studies have not provided critical evidence to demonstrate association between $T$. gondii and other feline pathogens [1].

\section{Conclusions}

The results of the present survey revealed a high seroprevalence of $T$. gondii infection in cats in Lanzhou, especially in stray cats. Knowing that cats play an important role in the transmission of $T$. gondii, revealing thus a significant public health concern, integrated strategies with efficient management measures should be taken to prevent and control T. gondii infection in cats in this city.

\section{Acknowledgements}

This work was supported, in part, by the National Natural Science Foundation of China (Grant Nos. 31172316 and 31101812), the Open Funds of the State Key Laboratory of Veterinary Etiological Biology, Lanzhou Veterinary Research Institute, Chinese Academy of Agricultural Sciences (Grant Nos. SKLVEB2009KFKT014, SKLVEB2010KFKT010 and

SKLVEB2011KFKT004) and the Yunnan Provincial Program for Introducing High-level Scientists (Grant No. 2009Cl125). The authors thank Dr J. P. Dubey, Animal Parasitic Diseases Laboratory, Animal and Natural Resources Institute, Beltsville Agricultural Research Center, United States Department of Agriculture, USA for providing the Toxoplasma gondii MAT antigen.

\section{Author details}

${ }^{1}$ State Key Laboratory of Veterinary Etiological Biology, Key Laboratory of Veterinary Parasitology of Gansu Province, Lanzhou Veterinary Research Institute, Chinese Academy of Agricultural Sciences, Lanzhou, Gansu Province 730046, PR China. ${ }^{2}$ College of Veterinary Medicine, South China Agricultural University, Guangzhou, Guangdong Province 510642, P R China. ${ }^{3}$ College of Animal Science and Technology, Yunnan Agricultural University, Kunming, Yunnan Province 650201, PR China. ${ }^{4}$ College of Veterinary Medicine, Gansu Agricultural University, Lanzhou, Gansu Province 730070, PR China.

Authors' contributions

$X Q Z$ and DHY conceived and designed the study, and critically revised the manuscript. SMW, DHZ and JC performed the experiments, analysed the data and drafted the manuscript. BQF, JFY, HQS and YBW helped in study 
design, study implementation and manuscript revision. All authors read and approved the final manuscript.

\section{Competing interests}

The authors declare that they have no competing interests.

Received: 3 October 2011 Accepted: 9 November 2011 Published: 9 November 2011

\section{References}

1. Dubey JP: Toxoplasmosis of Animals and Humans. CRC Press Inc., Boca Raton, New York; Second 2010, 1-313.

2. Zhou P, Chen Z, Li HL, Zheng H, He S, Lin RQ, Zhu XQ: Toxoplasma gondii infection in humans in China. Parasit Vectors 2011, 4:165.

3. Montoya JG, Liesenfeld O: Toxoplasmosis. Lancet 2004, 363:1965-1976.

4. Cook AJ, Gilbert RE, Buffolano W, Zufferey J, Petersen E, Jenum PA, Foulon W, Semprini AE, Dunn DT: Sources of Toxoplasma infection in pregnant women: European multicentre case-control study. $\mathrm{Br}$ Med J 2000, 312:142-147.

5. Zhao GH, Zhang MT, Lei LH, Shang CC, Cao DY, Tian TT, Li J, Xu JY, Yao YL, Chen DK, Zhu XQ: Seroprevalence of Toxoplasma gondii infection in dairy goats in Shaanxi Province, Northwestern China. Parasit Vectors 2011, 4:47.

6. Elmore SA, Jones JL, Conrad PA, Patton S, Lindsay DS, Dubey JP: Toxoplasma gondii: epidemiology, feline clinical aspects, and prevention. Trends Parasitol 2010, 26:190-196.

7. Dabritz HA, Conrad PA: Cats and Toxoplasma implications for public health. Zoonoses Public Health 2010, 57:34-52.

8. Cenci-Goga BT, Rossitto PV, Sechi P, McCrindle CM, Cullor JS: Toxoplasma in animals, food, and humans: an old parasite of new concern. Foodborne Pathog Dis 2011, 8:751-762.

9. Lee SE, Kim JY, Kim YA, Cho SH, Ahn HJ, Woo HM, Lee WJ, Nam HW: Prevalence of Toxoplasma gondii infection in stray and household cats in regions of Seoul, Korea. Korean J Parasitol 2010, 48:267-270.

10. Dubey JP, Zhu XQ, Sundar N, Zhang H, Kwok OC, Su C: Genetic and biologic characterization of Toxoplasma gondii isolates of cats from China. Vet Parasitol 2007, 145:352-356.

11. Zhang $H$, Zhou DH, Zhou P, Lun ZR, Chen XG, Lin RQ, Yuan ZG, Zhu XQ. Seroprevalence of Toxoplasma gondii infection in stray and household cats in Guangzhou, China. Zoonoses Public Health 2009, 56:502-505.

12. Lu AT, Gao Y, Du S: Survey of cats and dogs infected with Toxoplasma gondii in some areas of inner mongolia. Ani Husbandry Feed Sci 2010, 31:155-156, (in Chinese).

13. Xie GP, Geng YJ, Zhang RL, Huang DN, Gao ST: Survey of Toxoplasma gondii infection in pet cats and dogs in Shenzhen. Chin Trop Med 2010, 10:1075-1077, (in Chinese).

14. Dubey JP, Desmonts G: Serological responses of equids fed Toxoplasma gondii oocysts. Equine Vet J 1987, 19:337-339.

15. Seefeldt SL, Kirkbride CA, Dubey JP: Comparison of enzyme-linked immunosorbent assay, indirect fluorescent antibody test, and direct agglutination test for detecting Toxoplasma gondii antibodies in naturally aborted ovine fetuses. J Vet Diagn Invest 1989, 1:124-127.

16. Yan C, Yue CL, Yuan ZG, Lin RQ, He Y, Yin CC, Xu MJ, Song HQ, Zhu XQ: Molecular and serological diagnosis of Toxoplasma gondii infection in experimentally infected chickens. Vet Parasitol 2010, 173:179-183.

17. Wu SM, Huang SY, Fu BQ, Liu GY, Chen JX, Chen MX, Yuan ZG, Zhou DH, Weng YB, Zhu XQ, Ye DH: Seroprevalence of Toxoplasma gondii infection in pet dogs in Lanzhou, Northwest China. Parasit Vectors 2011, 4:64.

18. Akhtardanesh B, Ziaali N, Sharifi H, Rezaei S: Feline immunodeficiency virus, feline leukemia virus and Toxoplasma gondii in stray and household cats in Kerman-Iran: seroprevalence and correlation with clinical and laboratory findings. Res Vet Sci 2010, 89:306-310.

19. Zhang HC, Li PW, Cai JT: Survey of Toxoplasma gondii infection in pet cats and dogs in Zhengzhou. Heilongjiang J Ani Sci Vet Med 2010, 10:74-75, (in Chinese).

20. $\mathrm{Yu} Y \mathrm{YL}, \mathrm{Fu} L \mathrm{~L}$, Wang M: Serological survey of Toxoplasma gondii infection in dogs and cats in Beijing. Chin J Vet Med 2006, 42:7-9, (in Chinese).

21. Miró G, Montoya A, Jiménez S, Frisuelos C, Mateo M, Fuentes I: Prevalence of antibodies to Toxoplasma gondii and intestinal parasites in stray, farm and household cats in Spain. Vet Parasitol 2004, 126:249-255.
22. Haddadzadeh HR, Khazraiinia P, Aslani M, Rezaeian M, Jamshidi S, Taheri M, Bahonar A: Seroprevalence of Toxoplasma gondii infection in stray and household cats in Tehran. Vet Parasitol 2006, 138:211-216.

doi:10.1186/1756-3305-4-214

Cite this article as: Wu et al:: Seroprevalence of Toxoplasma gondii infection in household and stray cats in Lanzhou, northwest China. Parasites \& Vectors 2011 4:214

\section{Submit your next manuscript to BioMed Central and take full advantage of:}

- Convenient online submission

- Thorough peer review

- No space constraints or color figure charges

- Immediate publication on acceptance

- Inclusion in PubMed, CAS, Scopus and Google Scholar

- Research which is freely available for redistribution

Submit your manuscript at www.biomedcentral.com/submit
C Biomed Central 